\title{
Structural and Functional Analyses of Liver Cysts from the BALB/c-cpk Mouse Model of Polycystic Kidney Disease
}

\author{
Monalisa N. Muchatuta, * Vincent H. Gattone II, $†$ Frank A. Witzmann, $\$$ And \\ BONNIE L. BLAZER-YosT* ${ }^{\dagger},+, 1$ \\ *Department of Biology, Indiana University Purdue University at Indianapolis, Indianapolis, Indiana \\ 46202; †Department of Anatomy and Cell Biology, Indiana University School of Medicine, \\ Indianapolis, Indiana 46202; and $\$$ Department of Cellular \& Integrative Physiology, Indiana \\ University School of Medicine, Indianapolis, Indiana 46202
}

\begin{abstract}
Liver cysts arising from hepatic bile ducts are a common extrarenal pathology associated with both autosomal dominant and recessive polycystic kidney disease in humans. To elucidate the functional and structural changes inherent in cyst formation and growth, hepatic bile duct epithelia were isolated from the BALB/ c-cpk mouse model of polycystic kidney disease. Light and transmission electron microscopy revealed substantial fibrosis in the basal lamina surrounding hepatic bile duct cysts isolated from heterozygous (BALB/c-cpk/+) and homozygous (BALB/ccpk/cpk) animals. Scanning electron microscopy and length analysis of normal, precystic and cystic bile ducts provided the unique observation that primary cilia in cholangiocytes isolated from bile ducts and cysts of animals expressing the mutated cpk gene had lengths outside the minimal and maximal ranges of those in cells lining bile ducts of wild-type animals. Based on the hypothesis that PKD is one of several diseases characterized as ciliopathies, this abnormal variability in the length of the primary cilia may have functional implications. Electrophysiological analyses of freshly isolated cysts indicate that the amiloridesensitive epithelial $\mathrm{Na}^{+}$channel $(\mathrm{ENaC})$ is inactive/absent and cAMP-mediated anion secretion is the electrogenic transport process contributing to cyst fluid accumulation. Anion secretion can be stimulated by the luminal stimulation of adenylyl cyclase. Exp Biol Med 234:17-27, 2009
\end{abstract}

The electron microscopy studies were performed at the Indiana University School of Medicine EM Center, thanks to the generous support of that facility by the Polycystic Kidney Disease Foundation.

${ }^{1}$ To whom correspondence should be addressed at Biology Department, Indiana University Purdue University at Indianapolis, 723 West Michigan Street, Indianapolis, IN 46202. E-mail: bblazer@iupui.edu

Received July 3, 2008.

Accepted September 10, 2008.

DOI: $10.3181 / 0807-\mathrm{RM}-215$

$1535-3702 / 09 / 2341-0017 \$ 15.00$

Copyright (C) 2009 by the Society for Experimental Biology and Medicine
Key words: ciliary ion transport; primary cilia length; cyst wall electrophysiology; cholangiocytes

\section{Introduction}

Polycystic kidney disease (PKD) is a genetic disorder characterized by the growth of numerous fluid-filled cysts predominately in the kidney and liver. There are two forms of PKD which combined represent the third leading cause of kidney failure in the United States $(1,2)$. Autosomal Dominant Polycystic Kidney Disease (ADPKD), the most common form, occurs at a rate of approximately 1 in 800 in the human population and is usually diagnosed after the fourth decade of life. Autosomal Recessive Polycystic Kidney Disease (ARPKD) is a less common, but more severe, disease that is typically diagnosed in neonates and children. The primary cause of morbidity and mortality in both forms of PKD is the progression of renal pathology. The most common extra-renal manifestation of PKD is liver cysts which arise from the cholangiocytes lining the hepatic bile duct. As ARPKD progresses (adolescence to young adults), liver disease becomes an important and severe clinical finding. The cysts' size and associated fibrosis can affect organ function and quality of life. Most published research has been devoted to studying the mechanisms involved in renal cyst formation and comparatively little is known about the processes that control the development and maintenance of hepatic biliary cysts. This study addresses this gap by examining structural features of cyst formation as well as performing the first characterization of ion transport across freshly isolated cystic biliary tissue.

ADPKD arises from mutations in one of 2 genes, PKD1 or PKD2, coding for integral membrane glycoproteins, polycystin-1 and polycystin-2. The polycystins are found in multiple sub-cellular sites including the primary cilium 
located on the apical membrane of polarized cells. ARPKD arises from a mutation in PKHD1 which codes for another cilia-associated protein, fibrocystin (1-3). Most rodent models of PKD mimic the symptomatology of the disease while expressing mutations in proteins other than the polycystins or fibrocystin. Interestingly, the majority of the genetic defects in the rodent models, as well as other forms of cystic disease in humans, encode proteins that are found in the primary cilium (1-5).

The proposed functions of the primary cilium include roles in generation of left-right asymmetry during development, in control of cell cycle progression and cell proliferation, and in sensing and responding to, changes in fluid flow and/or tonicity (6-10). In PKD, alteration of any of these functions could theoretically be translated into mechanisms which would contribute to the progression and maintenance of fluid-filled cysts. In rodent models of PKD, diverse changes in cilia structure have been noted. These include shortened, malformed cilia as well as cilia which are unusually long or entirely absent (5). The correlation, if any, between ciliary structure and function in the context of PKD disease progression is limited.

Recent studies have indicated similarities associated with the development of kidney and liver cysts. Both processes involve barrier epithelial cells lining either the renal tubules or the biliary ducts, respectively. In these tissues, cAMP can act as a mediator of ion transporters involved in fluid homeostasis and as a mitogenic agent (1013). Emerging studies performed in both renal principal cells and biliary cholangiocytes link changes in flowinduced intracellular $\mathrm{Ca}^{2+}$ signaling, via the primary cilium, to changes in intracellular cAMP concentrations $(6,12,13)$.

The role of ion flux in the development of fluid-filled renal cysts is well documented. Elegant studies performed over a decade ago suggested that renal cyst formation in ADPKD is driven by anion secretion (14-17). Inhibitor studies and electrophysiological analyses indicated that CFTR (cystic fibrosis transmembrane regulator), located in the apical membrane, may be the ultimate $\mathrm{Cl}^{-}$channel responsible for the secretion while the $\mathrm{Na}^{+} \mathrm{K}^{+} 2 \mathrm{Cl}^{-}$triple cotransporter, located in the basolateral membrane, is responsible for the influx of $\mathrm{Cl}^{-}$into the epithelial cells. Ye and Grantham (14) isolated renal cysts from ADPKD patients and measured net fluid movement under control and forskolin stimulated conditions. Under unstimulated conditions the cysts behaved as absorptive epithelia. The fluid movement was reversed by forskolin, an activator of adenylyl cyclase which increases intracellular cAMP and, presumably, ion transport via CFTR. These findings were extended by Grantham et al. to demonstrate that primary cultures of cells isolated from human cysts also responded to forskolin with an increase in fluid secretion into the cyst lumen and that the fluid contained endogenous secretogogues that could mimic the effect of forskolin $(14,16,18)$. The latter study indicates that, while stimulus-coupled anion secretion may play a role in cyst formation and main- tenance, the cyst fluid may contain additional contributing factors.

Much less is known about the ion transport processes that contribute to the formation and maintenance of the hepatic biliary cysts. CFTR is present and active in bile duct epithelial cells $(19,20)$. A single report has delineated the ion transport characteristics of cultured liver cyst epithelial cells (21). These investigators isolated epithelial cells from the liver cysts of two different murine models of PKD, grew the cells to confluence, and used electrophysiological techniques to characterize the ion transport properties of the resulting high resistance epithelial monolayers. Amiloride sensitive absorptive $\mathrm{Na}^{+}$flux was present in the cultured cells indicating the presence of the epithelial $\mathrm{Na}^{+}$channel $(\mathrm{ENaC})$. In addition, there was a substantial $\mathrm{Cl}^{-}$secretory response that was responsive to agonists that elevated intracellular cAMP (21). The anion secretory response had the characteristics of CFTR-mediated transport. In contrast, Kirkland and colleagues found no amiloride sensitivity in isolated gallbladder epithelia (22). To our knowledge, there have been no studies examining the transport characteristics of freshly isolated hepatic cyst wall epithelia.

The current studies have been performed to analyze fibrotic changes, ciliary structure and transepithelial ion flux during hepatic cyst development in the BALB/c-cpk mouse model of PKD. The homozygous BALB/c-cpk/cpk animals die of renal disease before maturity while the heterozygous $\mathrm{BALB} / \mathrm{c}-\mathrm{cpk} /+$ animals live to breeding age and after $12-18$ months express biliary hepatic cysts which are similar to those found in human ADPKD patients (23).

The initiation step for cyst formation is not completely understood; however, there are three main components that are consistently present during the process in both renal and hepatic cysts: cellular proliferation, fluid accumulation and structural changes to the extra-cellular matrix around the cysts. To begin to understand these processes we have compared the ultra-structural characteristics of the liver cysts with normal bile duct epithelia to identify the degree of cellular proliferation and fibrosis surrounding the cyst epithelia. Electron microscopy and measurement of cilia length were used to compare the primary cilia of cyst wall epithelial cells with those found in normal and pre-cystic bile ducts. Functionally, the characteristics of the transepithelial ion transport in response to increases in intracellular cAMP were measured in freshly isolated cyst wall epithelia.

\section{Materials and Methods}

Mice. The cpk gene is expressed primarily in liver and kidney and encodes the protein "cystin". BALB/c mice homozygous for a $c p k$ mutation $(\mathrm{BALB} / \mathrm{c}-\mathrm{cpk} / \mathrm{cpk})$ rapidly develop polycystic kidney disease with the expression of a multi-organ phenotype and die within 2-4 weeks (23). Heterozygous animals (BALB/c-cpk/+) have a relatively normal life span and can be used for breeding. As 

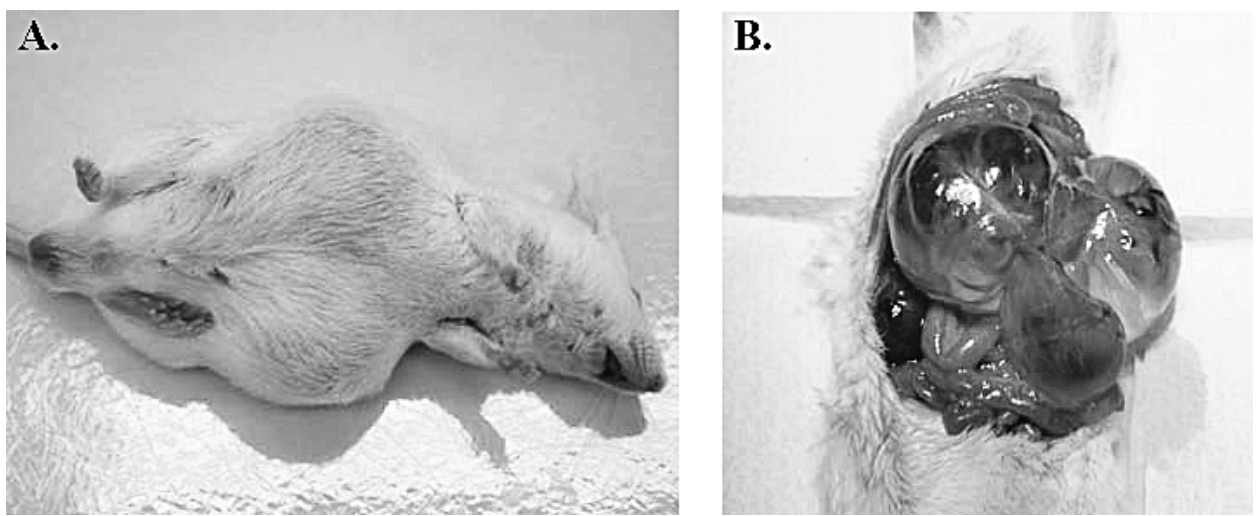

Figure 1. Photographs of an 18 month old male BALB/c-cpk/+ mouse showing an increase in abdominal girth (A) due to the presence of extensive liver cysts (B).

heterozygote mice age, they begin to develop liver cysts which are phenotypically similar to hepatic biliary cysts observed in human ADPKD. The cysts range in size from microscopic to the large fluid-filled masses seen in Figure 1. After 12-18 months of age, these animals express multiple large fluid-filled hepatic cysts that arise from the intrahepatic biliary system. The mice were bred at the Indiana University School of Medicine laboratory animal resource center and used under protocols approved by IACUC.

Electrophysiological Analysis of Ion Transport in Isolated Liver Cyst Epithelia. Normal and cystic BALB/c mice ( $\sim 18$ months of age) were euthanized (150 $\mathrm{mg}$ pentobarbital/Kg body weight) and perfused with phosphate buffered saline (PBS). Large liver cysts from $\mathrm{BALB} / \mathrm{c}-\mathrm{cpk} /+$ mice were dissected free from surrounding hepatic tissue, opened by peripheral cuts to form a single sheet and mounted in a modified Ussing chamber. Cysts used for electrophysiological measurements had tissue sheets large enough to obtain a circular area of at least 1.3 $\mathrm{cm}$ in diameter. During the electrophysiological experiments, the cystic tissue was bathed in serum-free Dulbecco's modified Eagles medium/F12 media (DMEM) (Invitrogen, Grand Island, NY), supplemented with 2.4 $\mathrm{mg} / \mathrm{L}$ sodium bicarbonate (Fisher Scientific, Fair Lawn, NJ), and $2 \mathrm{mM}$ glutamine. The media was maintained at $37^{\circ} \mathrm{C}$, with gentle circulation provided by a $5 \% \mathrm{CO}_{2}-95 \% \mathrm{O}_{2}$ gas lift. Electrodes inserted into the bathing media on either side of the tissue allowed for the measurement of the spontaneous transepithelial potential difference (PD). Using a current/voltage clamp, the PD was clamped to zero and the resulting short circuit current (SCC), a measure of net transepithelial ion transport, was recorded continuously. The epithelial layers were monitored until a steady basal current was obtained (20-60 mins). Effectors (from 1000X stock) were added to the apical and/or serosal bathing medium as indicated in the figures and the ion transport responses of the tissue were measured. Agonist/inhibitors and final concentrations: Forskolin, $5 \mu \mathrm{M}$, is an activator of adenylyl cyclase; 5-nitro-2-(3-phenylpropylamino)-benzoic acid
(NPPB), $100 \mu \mathrm{M}$, is a $\mathrm{Cl}^{-}$channel blocker; and amiloride, $10 \mu \mathrm{M}$, is a specific inhibitor of $\mathrm{ENaC}$.

Light and Electron Microscopy (EM). Cysts were excised from the livers of the animals, immediately placed in EM fixative (2\% glutaraldehyde, $2 \%$ paraformaldehyde, $100 \mathrm{mM}$ phosphate buffer, $\mathrm{pH}$ 7.4) and fixed overnight at room temperature. The next day cysts were cut into smaller pieces and returned to the EM fixative before embedment in Epon812 resin for evaluation by light and electron microscopy.

Light microscopy of $1 \mu \mathrm{m}$ thick sections was used to analyze structural differences and similarities between normal and cystic tissue epithelia, and to provide an overview of the area to be viewed using electron microscopy. Electron microscopy was performed to analyze the structural differences between the cystic epithelial cells in contrast to normal bile duct cholangiocytes. For transmission electron microscopy (TEM), tissues were post-fixed in $1 \%$ osmium tetroxide for $1 \mathrm{hr}$, rinsed, and dehydrated in graded ethanol solutions and propylene oxide resin of 1:3, 1:1 and 3:1 and left overnight before being embedded in Epon 812 and allowed to polymerize at $60^{\circ} \mathrm{C}$ for $24 \mathrm{hrs}$. Semi-thick $(1 \mu \mathrm{m})$ resin sections were cut and stained with Toluidine blue and visualized with a light microscope. Thin sections $(60 \mathrm{~nm} / 80 \mathrm{~nm})$ were cut, stained with uranyl acetate and lead citrate and visualized on a Tecnai G12 TEM (FEI, Hillsboro, Oregon).

Samples to be visualized using scanning electron microscope (SEM) were rinsed in PBS, incubated in $1 \%$ osmium tetroxide for $1 \mathrm{hr}$, rinsed in distilled water, dehydrated in graded ethanol solutions and then dried using a Tousimis Samdri 790 critical point drier, with liquid $\mathrm{CO}_{2}$ as the transitional fluid. After drying, samples were mounted onto stubs, coated with a gold/palladium $(\mathrm{Au} / \mathrm{Pd})$ by a Polaron direct sputter coater and viewed with a JEOL JSM 6390 scanning EM (JEOL USA Inc., Peabody, MA). Micrographs were taken at appropriate magnifications. Cilia length was determined from the SEM micrographs with the aid of Scandium program (Sift Imaging Systems, Lakewood, $\mathrm{CO}$ ). 

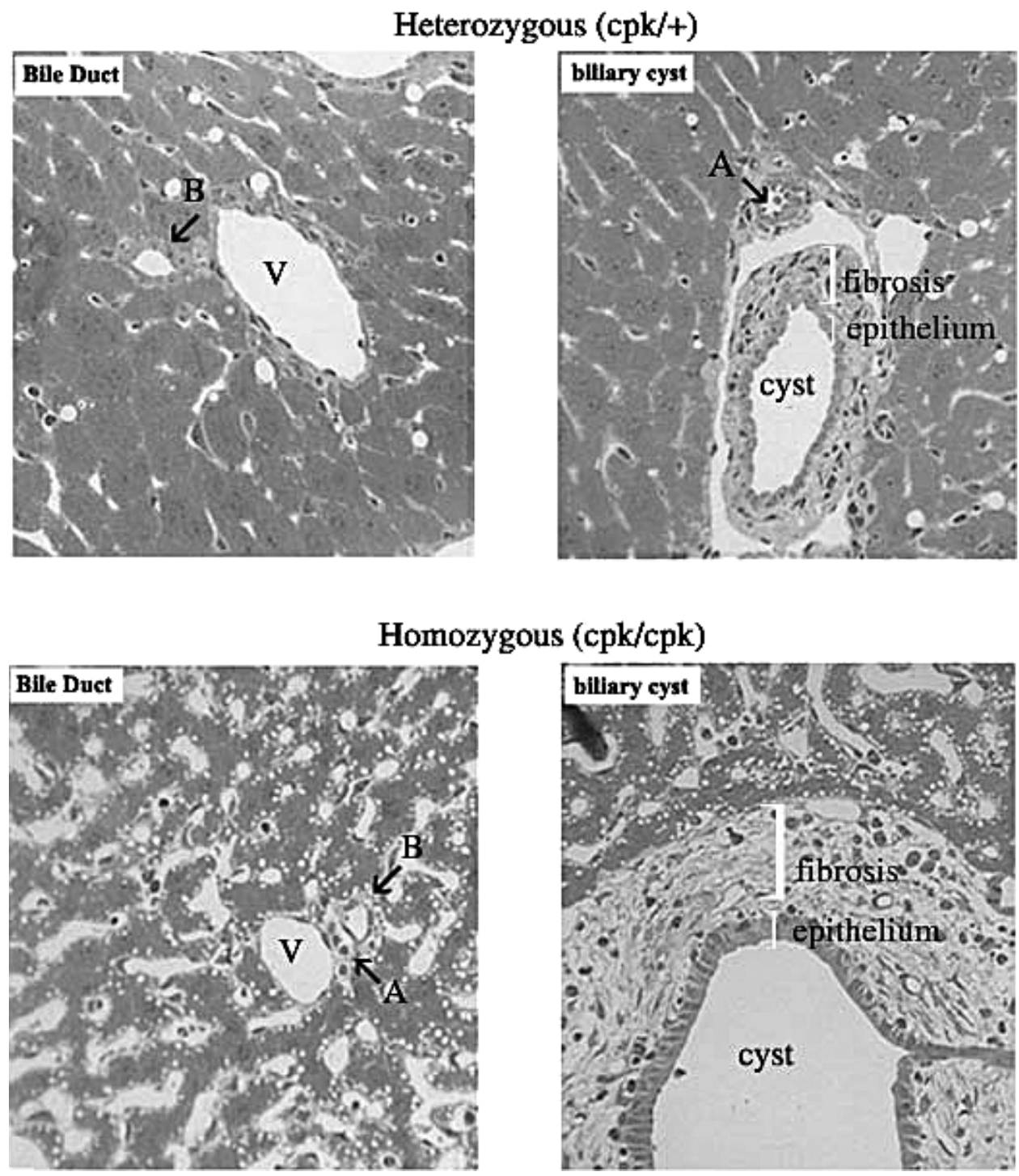

Figure 2. Light microscopic images of liver sections showing normal and cystic bile ducts of heterozygous (BALB/c-cpk/+) and homozygous (BALB/c-cpk/cpk) mice. All images were taken with a $\times 40$ objective. The bile ducts/cysts are generally closely associated with the portal triads (bile duct-B, portal vein-V and hepatic artery branch-A). The cystic bile ducts (right) from both the cpk/+ heterozygotes (top) and cpk/cpk homozygotes (bottom) are associated with an increase in periportal fibrosis.

\section{Results}

At approximately $1-1.5$ years of age BALB/c-cpk/+ mice (male/female) exhibit abdominal distention as a result of the expansion of the hepatic cysts (Fig. 1A). There are multiple cysts of varying sizes in the hepatic biliary tree (Fig. 1B).

\section{Structural Comparisons of Normal and Cystic} Hepatic Bile Ducts. At the light microscope level, a comparison of normal appearing bile ducts with hepatic biliary cysts, which are formed from the same cell type, clearly shows the cellular proliferation necessary for the increase in luminal diameter that takes place during cyst formation (Fig. 2). The number of epithelial cells lining the cysts is several fold more than the cells lining the normal bile duct. Another striking aspect of the cysts is the amount of fibrosis associated with the cystic epithelia.
Transmission electron microscopy (TEM) images of normal appearing bile ducts from wild-type, heterozygous and homozygous mice are shown in Figure 3. In general, the majority of the bile ducts in mice carrying one or two copies of the aberrant cpk gene are enlarged. Even those that appear relatively normal in size likely represent the "precystic" state, a supposition that is supported by the homozygous bile duct in Figure 3 where cell division is occurring even though the bile duct is lined with a confluent layer of epithelial cells. Interestingly, the cell that is dividing in the upper portion of the duct appears to be separating in a circumferential direction which may be indicative of loss of planar cell polarity, a defect seen in models of PKD.

Scanning electron microscopic (SEM) analysis reveals the primary cilia found in both the bile ducts and in the cysts of affected animals (Fig. 4). The representative micrographs 


\section{Bile Ducts}

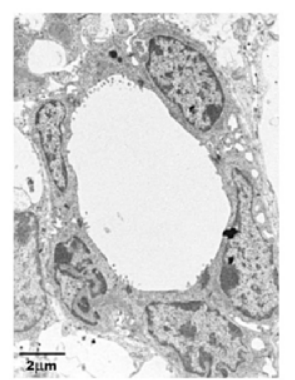

Adult Wild Type

$(+/+)$

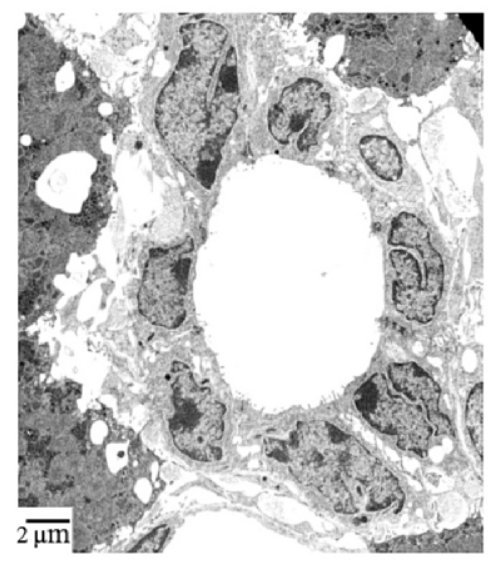

Heterozygous

(cpk/+)

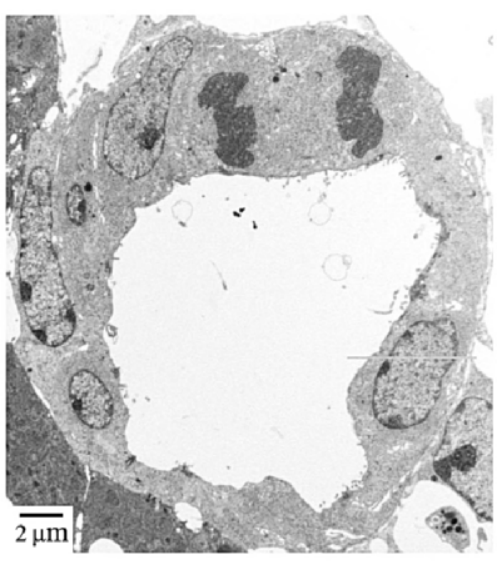

Homozygous

(cpk/cpk)

Figure 3. TEM of bile ducts/cysts from BALB/c-cpk mice. The relatively normal sized bile ducts in adult heterozygous mice (center) and suckling homozygous mice (right) are lined by cuboidal epithelial cells similar in appearance to those seen in adult normal (wild-type, $+/+)$ mice. However the ducts from these cystic mice generally have more cells per duct cross-section and exhibit evidence of increased proliferative activity (mitosis in panel on the right). All figures are the same magnification.

of cystic epithelia show large differences in ciliary length within the same cyst. To provide a more quantitative analysis of potential ciliary differences, the length of the cilia from multiple animals were measured and the data are expressed as a percentage of the total in various length classifications. The category of lengths from 1-4 $\mu \mathrm{m}$ contains $93 \%$ of the wild-type cilia (Fig. 5). In general, many of the epithelial cells in bile ducts and cysts from heterozygous and homozygous animals possessed cilia that were either shorter or longer than those observed in bile ducts from wild-type animals.

Careful examination of SEM micrographs identified structural differences between certain epithelia isolated from the same liver cyst (compare Fig. 6A and B). In one area (Fig. 6A) epithelia had a domed appearance accompanied by numerous long cilia. However, another area in the same cyst possessed cells with a flattened phenotype accompanied by very short or absent cilia (Fig. 6B).

Ion Transport Properties of Cyst Epithelia. Electrophysiological experiments were performed on isolated cyst wall epithelia from the largest of the biliary liver cysts (Fig. 7). The cysts are formed of a low resistance epithelial layer (all 5 experimental tissues have resistances between 30 and 40 $\Omega \mathrm{cm}^{2}$ ). The cholangiocytes which line the cysts showed electrogenic ion secretion in response to forskolin, an agent known to increase intracellular cAMP. The first panel (Fig. 7A) illustrates a response when forskolin was added to both the luminal and serosal sides of the epithelia. The increase in SCC represents either an increase in cationic movement in an absorptive direction (luminal to basal) or anionic movement in a secretory direction (basal to luminal). In contrast to most other epithelial cells where adenylyl cyclase (the protein target activated by forskolin) is associated with receptors on the basolateral membrane, forskolin was ineffective when added to the serosal face of the monolayer but was always effective when added to the apical side (Fig. 7B, representative of 5 out of 5 experiments).

Inhibitors of ion channels previously allied with transport in renal cysts were used to determine the nature of the SCC. Ion transport was not inhibited by amiloride, a specific ENaC blocker, indicating that $\mathrm{Na}^{+}$reabsorption does not contribute to basal or forskolin stimulated ion transport. 5-nitro-2-(3-phenylpropylamino)-benzoic acid (NPPB), a $\mathrm{Cl}^{-}$channel blocker, inhibited trans-epithelial transport but only when added to the apical side of the epithelial sheet indicating that in the cholangiocytes, like the renal cells, the anion channels are in the apical membrane. Based on data suggesting a role for CFTR in renal cystogenesis, it is likely that the chloride channel involved in the biliary cysts is also CFTR.

The two experiments shown in Figure 7 are representative of 5 such experiments. In all 5, the absence of amiloride sensitivity was consistent. The figures shown were selected to illustrate the variability in basal transport rates (from 7-40 $\mu \mathrm{A} / \mathrm{cm}^{2}$ ) as well as the differences in the percentage of the final current which was inhibited by NPPB.

\section{Discussion}

Treatment of PKD requires an understanding of the 


\section{Bile Ducts}

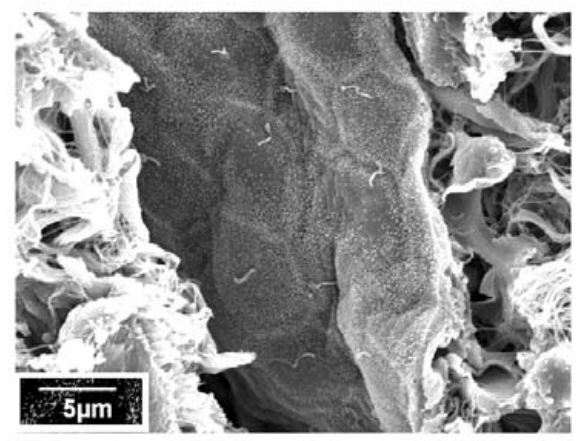

Wild Type (+/+)

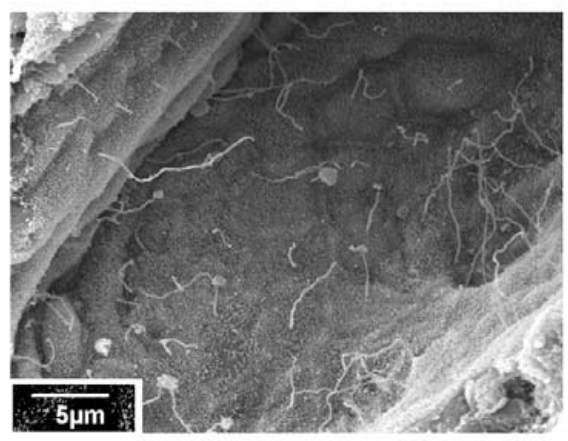

Heterozygote (cpk/+)

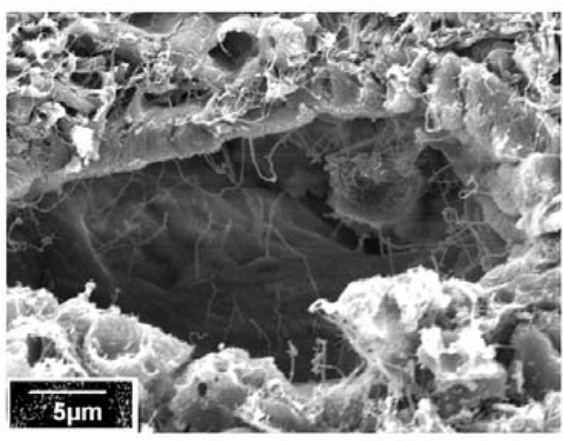

Homozygote (cpk/cpk)

\section{Cysts}

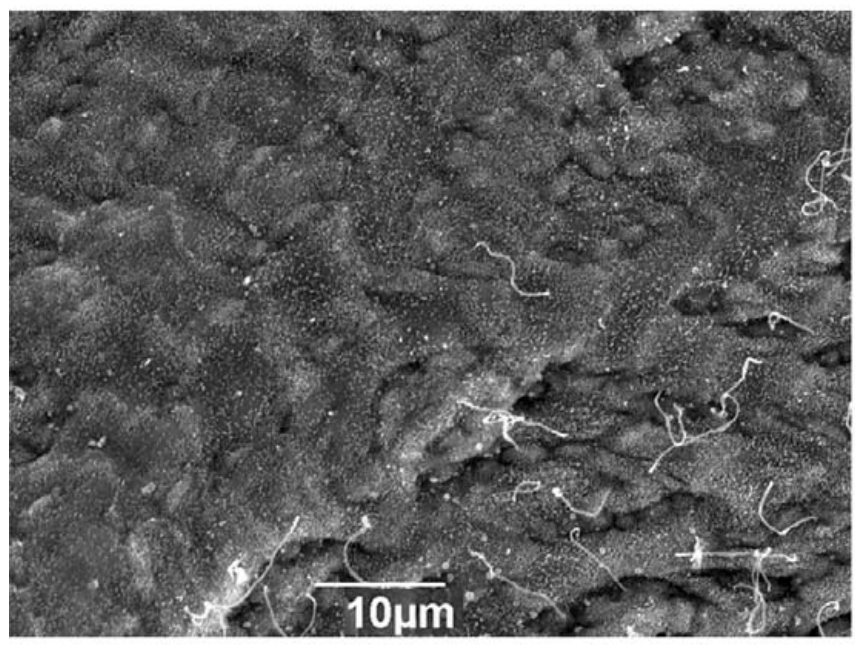

Heterozygote $(\mathrm{cpk} /+)$

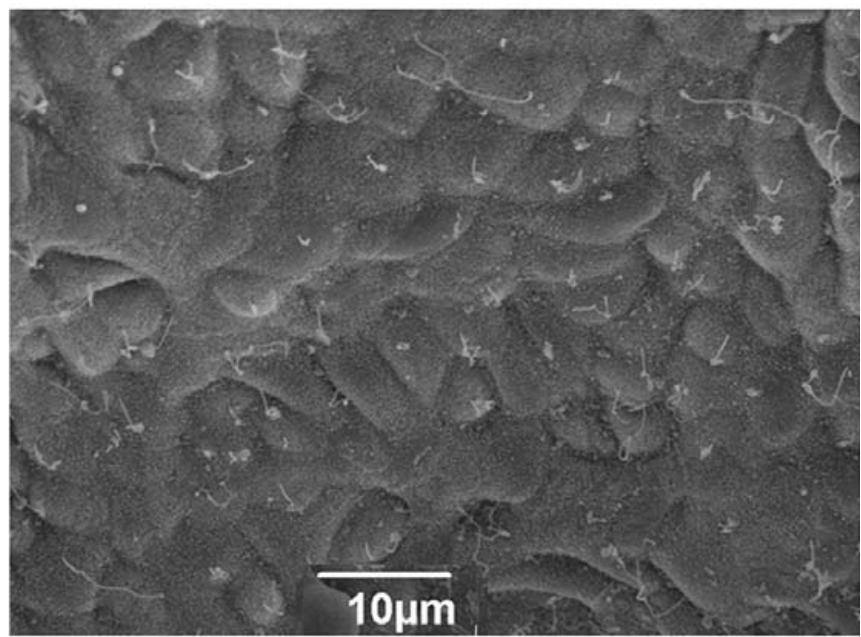

Homozygote (cpk/cpk)

Figure 4. SEM of bile ducts/cysts from BALB/c-cpk mice. The micrographs of bile ducts from normal, heterozygous-cpk/+ and homozygous$\mathrm{cpk} / \mathrm{cpk}$ mice (top micrographs) all possess ciliated epithelia; however, the cilia in the normal mice all appear roughly the same length while cilia in heterozygous and homozygous mice are quite variable in length. In the cysts from heterozygous and homozygous mice (bottom micrographs), the variability in cilia length is very obvious, from being almost non evident to cilia that are very long.

processes involved in the development and maintenance of the renal and extra-renal cysts. One of the most promising leads in the search for therapeutic agents to treat the renal pathology of both the autosomal dominant and recessive forms of polycystic kidney disease is the vasopressin V2 receptor antagonist OPC31260. In three different animal models of renal cystic disease, treatment with the antagonist decreased cAMP levels, prevented renal enlargement, inhibited cystogenesis and protected renal function by halting progression of renal disease $(24,25)$. Development of biliary cysts may share common features with the biochemical processes involved in renal cyst formation and this information can be used to develop similar treatment strategies for hepatic cystic disease. In both cases cyst formation involves the epithelial cells lining the renal tubule or the cholangiocytes of the intrahepatic bile ducts. Previous studies combined with the current data show that both may involve cAMP-mediated ion transport.

The BALB/c-cpk/cpk mouse is a model of ARPKD which expresses renal as well as extra-renal pathology. The homozygous affected mice live only $2-4$ weeks. However, the heterozygous animals (BALB/c-cpk/4) used for breeding 

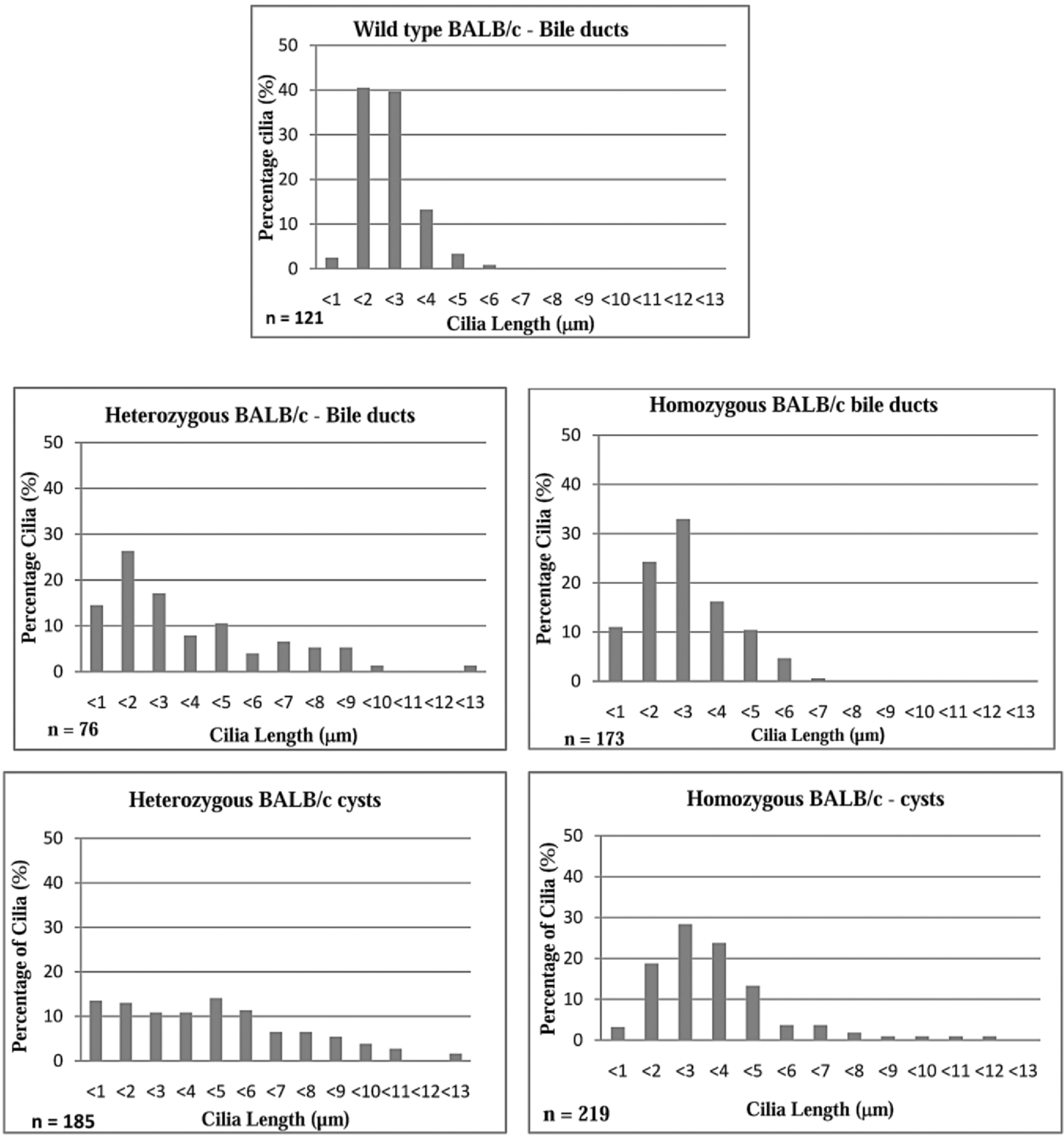

Figure 5. Cilia length distribution. Length was determined for the following numbers of cilia in each category: Wild-type, 121; heterozygous bile ducts, 76; homozygous bile ducts, 173; heterozygous cysts, 185; homozygous cysts, 219 . The bars represent the percentages of the total within each of the length categories indicated.

show some interesting pathology. Older heterozygous mice (12-18 months) develop intra-hepatic bile duct cysts which are phenotypically similar to the multiple, large, hepatic biliary cysts observed in human ADPKD (23). The phenotypic presentation of the liver cysts in this model is dominated by extracellular fibrosis, particularly in the tissues immediately surrounding the cysts. Our results are similar to Hou et al., who reported extracellular fibrosis surrounding the renal cysts in C57BL/6J model of PKD (3). Proliferative changes may be enhanced by the presence of proto-oncogenes in PKD (26).

Structural and functional differences in the primary cilium have been implicated in both renal and hepatic cystic disease. The primary cilium, a ubiquitous structure, particularly on polarized epithelial cells, contains a surpris- 

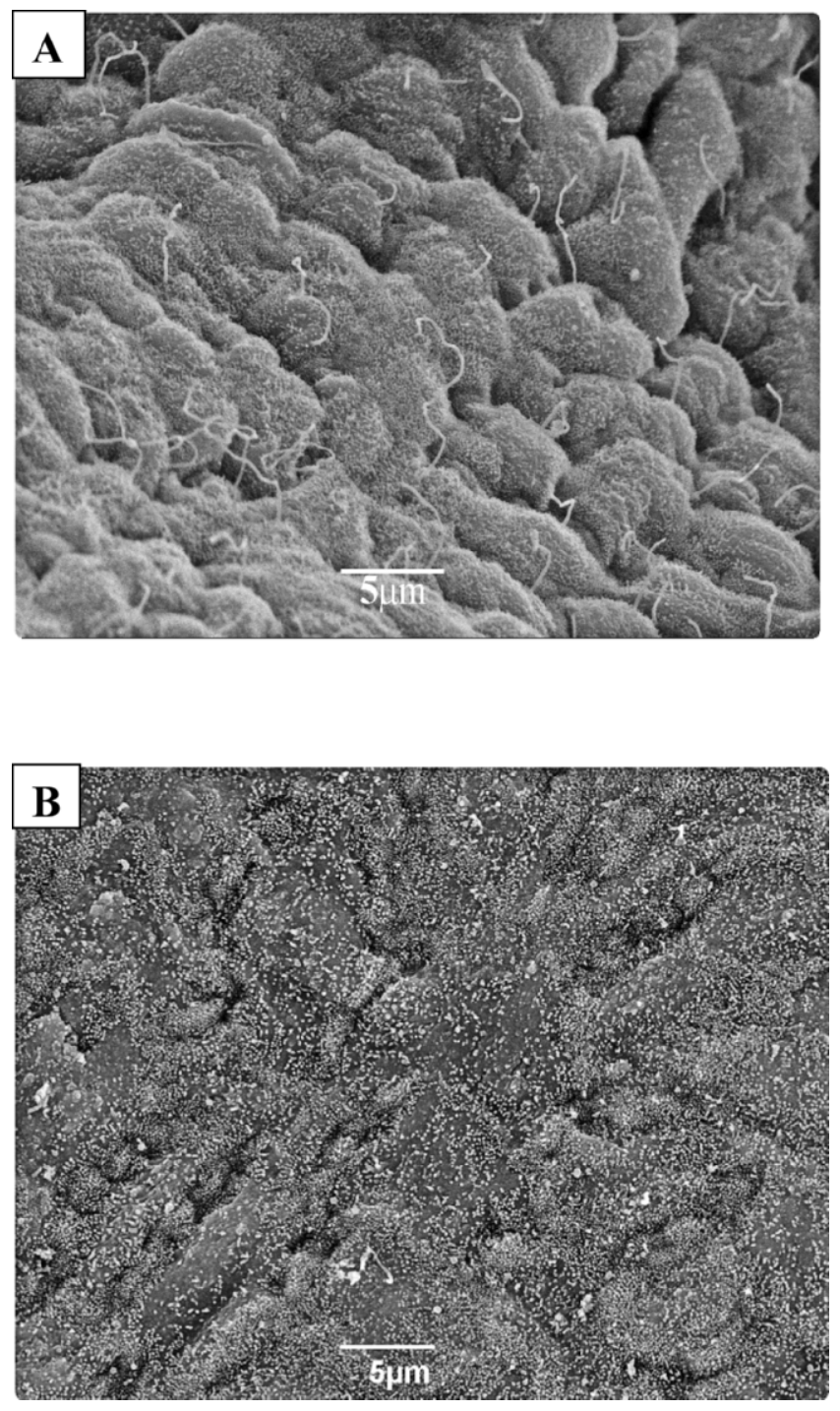

Figure 6. Ciliated and non-ciliated cholangiocyte cellular morphology. A. This micrograph depicts an area of a large cyst isolated from a BALB/c-cpk/+ mouse that contains predominately ciliated cholangiocytes expressing central cilia with lengths longer than those found in normal, wild-type bile ducts. This micrograph was obtained from the same cyst as that shown in panel B. B. This micrograph depicts an area of a large cyst isolated from a BALB/c-cpk/+ mouse that contains predominately non-ciliated cholangiocytes that express central cilia with lengths shorter than those found in normal, wild-type bile ducts. This micrograph was obtained from the same cyst as that shown in panel $A$.

ingly large number of proteins $(27,28)$. Of note are the findings that the majority of the proteins known to be involved in human and mammalian PKD are co-localized to this single large apical appendage or to the basal body at the cilium-cellular interface $(5,29)$. Exogenous expression studies of the cpk gene, whose mutation results ARPKD, showed the cpk gene product to be localized to the central cilia in polarized renal epithelia. Interestingly, another ciliaassociated protein, Polaris, also co-localizes with cystin in the cilia (3). These findings suggest that the functionality of the cilia plays a major role in cystogenesis. There are other disease entities which exhibit mutations in proteins that reside in the primary cilium and many of these diseases also express cyst formation in kidney, liver and other organs. The most notable of these diseases are Nephronophthsis (NPHP), Meckel-Gruber Syndrome and Bardet-Biedl Syndrome. In the latter, a mutation in one of a complex of trafficking proteins of the cilium causes a wide range of organ dysfunction $(4,30)$.

Recently Masyuk and colleagues (31) have convincingly characterized the cholangiocyte cilium as a sensory organelle which can detect changes in luminal bile duct flow. Changes in flow are communicated to other cellular response elements via changes in intracellular $\mathrm{Ca}^{2+}$ and cAMP concentrations. Increased flow causes a ciliumdependent rise in intracellular $\mathrm{Ca}^{2+}$ followed by a decrease in cAMP via a $\mathrm{Ca}^{2+}$ inhibitable adenylyl cyclase (AC6) which, interestingly, is also localized within the cilium (6). The apical localization of AC6 is entirely consistent with the apical effectiveness of forskolin, an adenylyl cyclase activator in the excised cyst epithelium. These data also suggest that components present in cyst fluid that bathes the apical surface may modulate electrolyte and compensatory fluid secretion into the cyst.

Since the primary cilium is thought to act as both a flow sensor and, potentially, an effector of ion transport, changes in ciliary structure may accompany important, as of yet unknown, alterations in ciliary function. In rodent models of PKD, diverse changes in ciliary structure have been previously noted. These include shortened, malformed cilia, multiciliated epithelial cells, as well as cilia which are unusually long or entirely absent (5). The reason for apparent discrepancies in the various models could result from inherent variability due to the fact that the underlying mutations are quite different. However, in the current experiments, we have noted that in both bile ducts and liver cysts there is a range of ciliary length, both shorter and longer, compared to those seen in wild-type ductal epithelia. For example, in heterozygous animals, there is an increase in the percentage of cilia that are less than $1 \mu \mathrm{m}$ in length$15 \%$ in bile ducts and $14 \%$ in liver cysts as compared to $2 \%$ in wild-type controls. This trend is also seen in homozygous animals where $11 \%$ of bile duct cilia and $3 \%$ of cilia from cystic epithelia are less than $1 \mu \mathrm{m}$ in length.

Cilia on epithelial bile duct cells in wild-type mice fall predominately within the range of $1-4 \mu \mathrm{m}$ with none of the 121 cilia counted having a length over $6 \mu \mathrm{m}$. This is in excellent agreement with the studies of Brown and Murcia who showed that in normal mouse renal cells, the majority of the cilia were $1-2 \mu \mathrm{m}$ with no cilia over $4 \mu \mathrm{m}$ in length (9). In contrast $20 \%$ of the cilia in heterozygous mice bile ducts, $26 \%$ in heterozygous cysts and $6 \%$ of cilia in homozygous mice cysts were over $6 \mu \mathrm{m}$. Given the finding that cystic cholangiocytes have both shorter and longer cilia than are seen in normal cholangiocytes, the mean length is less important than is the length distribution in the characterization of ciliary abnormalities. The relative 
A

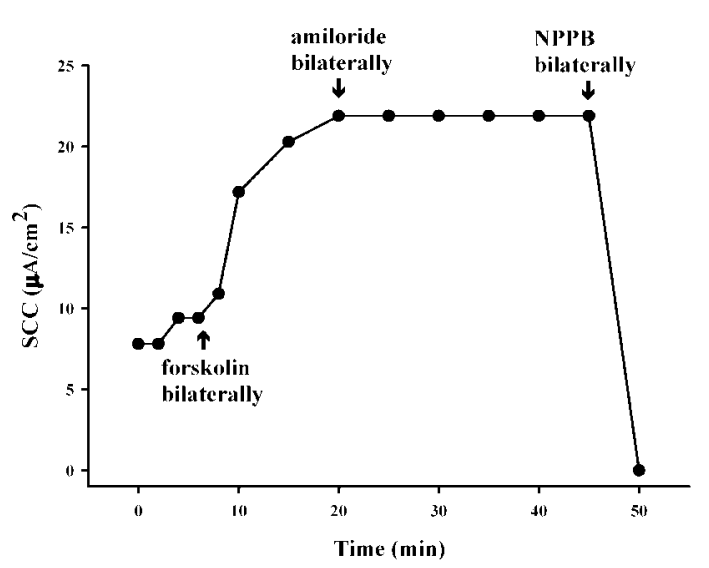

B

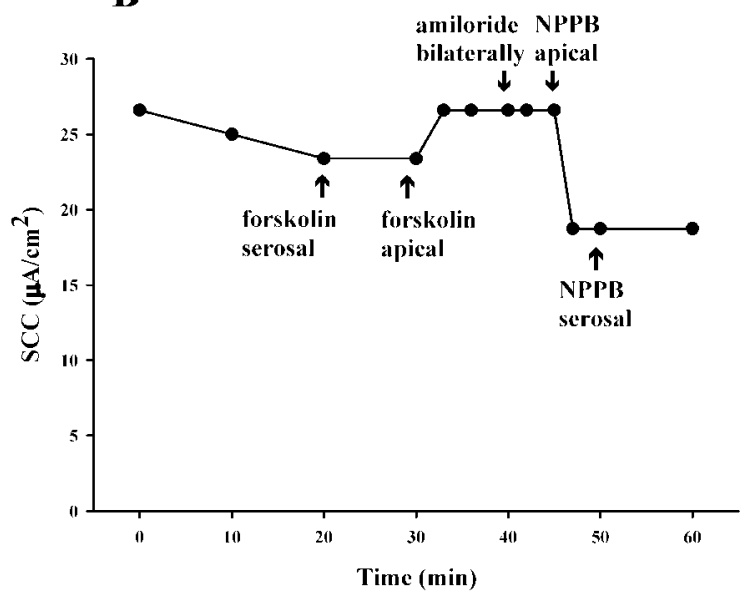

Figure 7. Electrophysiology of biliary epithelia lining the liver cysts found in BALB/c-cpk/+ mice. The epithelium in each panel represents a large liver cyst (each from a different mouse). Tissue was mounted in Ussing chambers and maintained under voltage clamp conditions at $37^{\circ} \mathrm{C}$. SCC, the short circuit current, is a measure of net transepithelial, electrogenic ion transport. Panel B shows the specific sidedness of the agonists/ inhibitors applied to the cyst epithelia. Effectors were added to the bathing media to achieve final concentrations of: amiloride, $10 \mu \mathrm{M}$; forskolin, 5 $\mu \mathrm{M} ; \mathrm{NPPB}, 100 \mu \mathrm{M}$.

similarities between the cilia length distribution in heterozygous and homozygous animals may relate to the possible loss of function of the normal cpk allele in the cells lining the cysts of the heterozygous mice whereby they express only the mutant cpk allele (32).

One possible explanation for the preponderance of very short or absent cilia is that the cystic and pre-cystic epithelia are strongly proliferative. The primary cilium is grounded in one of the two basal bodies which become centrioles needed during mitosis. Hence, in dividing cells, there is ciliary resorption and shortening during the $\mathrm{G} 2 / \mathrm{M}$ phase of the cell cycle (7). The excess of longer than normal cilia is intriguing. More studies will be necessary to determine whether this is a cause or consequence of cyst formation. However, the large number of very long cilia, particularly in the bile ducts of the heterozygous animals, suggests that the length may contribute to the development of cysts.

Sheets of liver cyst wall can be successfully isolated from heterozygous animals and used in electrophysiological studies to examine electrogenic transepithelial transport characteristics. The cholangiocytes that line the biliary duct cysts are responsible for the secretion of ions and fluid into the cyst cavity. Therefore, a careful characterization of the ion transport proteins and an elucidation of some of the intracellular biochemical pathways controlling the activity of the channels forms an important background for understanding cyst development and maintenance. The basal level of electrogenic ion flux in the isolated cholangiocyte-lined tissues showed considerable variation. This variation is unlikely to be due to the size of the cysts since only the largest of these could be used for electrophysiological experiments. Additional experiments will be necessary to determine if the differences are the result of factors such as cilia length, and/or level of transport protein abundance.

In contrast to cultured epithelia from cyst walls (21), the freshly isolated cystic epithelia were unresponsive to amiloride, a specific inhibitor of the epithelial $\mathrm{Na}^{+}$channel, $\mathrm{ENaC}$. These results suggest that electrogenic $\mathrm{Na}^{+}$reabsorption does not contribute to basal or forskolin-stimulated ion transport ex vivo and possibly in vivo. The differences between the amiloride sensitivity in the cultured cells versus the freshly isolated tissue may be explicable, in part, by the conditions necessary to grow cells in primary culture. These conditions generally include culture media with high levels of steroids (dexamethasone), growth factors (epidermal growth factor), pituitary extract, triiodothyrodine, insulin and forskolin-all factors that are known to regulate $\mathrm{ENaC}$ synthesis and/or activity. Alternatively, it has been suggested that the state of differentiation may change over time in primary culture (33).

NPPB inhibits transepithelial transport but only when added to the apical side of the epithelial sheet. NPPB inhibits multiple transport proteins; however, it is often used as an inhibitor of CFTR. The inhibition on the apical side is consistent with the expected apical localization of the CFTR $\mathrm{Cl}^{-}$channel where it can secrete anions into the cyst lumen.

Forskolin, an agent that activates adenylyl cyclase and thereby increases intracellular cAMP concentrations, increases net electrogenic transport with a directionality that is consistent with an increase in either cation absorption or anion secretion. Because the process is inhibited by NPPB but not amiloride, forskolin is likely causing an increase in $\mathrm{Cl}^{-}$secretion. There is, however, one unusual observation in these experiments. The forskolin is effective when added to the apical membrane. In many epithelial cells, adenylyl cyclase is localized to the serosal compartment where it is in contact with peptide hormone receptors resident in the basolateral membrane. For this reason, forskolin is usually effective from the serosal bathing media, not the apical. Our data are, as noted above, consistent with the recent observation by Masyuk et al. that adenylyl cyclase 6 is 
found in cholangiocyte primary cilia on the apical surface (6). As such, this enzyme is a target for agents that increase anion secretion. Alternatively, the supporting, fibrous membranes surrounding the basolateral surface of the epithelial monolayers may form a barrier for the diffusion of the forskolin.

Our studies have, for the first time, demonstrated the ion transport characteristics of freshly isolated bile duct cystic epithelia. The results indicate the remarkable similarity between the biliary and renal cystic tissue. However, the absence of vasopressin, V2, receptors in cholangiocytes means that, unlike the renal principal cells, antidiuretic hormone will not be an endogenous ligand stimulating increases in intracellular cAMP. Thus, the V2 receptor antagonists will not be effective for treating the hepatic manifestations of PKD. However, in the PCK rat model of ARPKD, octreotide, an analogue of somatostatin appears to be effective in inhibiting cAMP levels and reducing cyst growth (34). Our data are consistent with this finding and provide additional support for the proposed mechanism of drug action.

In summary, our results indicate that the cholangiocytes lining the hepatic bile ducts exhibit a diverse range of primary cilia lengths, which are outside the normal minimal and maximal ranges in wild-type animals. This may contribute to the observed differences in cellular morphology and/or cellular ion transport observed in the cystic epithelia. With regard to ion transport, our studies are the first to use ex vivo tissue to demonstrate ion secretory flux, which is consistent with cyst enlargement via compensatory fluid movement. The observation that apical activation of adenylyl cyclase is a stimulus for ion flux suggests that the components of the cyst fluid may contribute to cyst enlargement.

1. Torres VE, Harris PC. Mechanisms of disease: autosomal dominant and recessive polycystic kidney diseases. Nat Clin Pract Nephrol 2:40-55; quiz 55, 2006.

2. Torres VE, Harris PC. Polycystic kidney disease: genes, proteins, animal models, disease mechanisms and therapeutic opportunities. J Intern Med 261:17-31, 2007.

3. Hou X, Mrug M, Yoder BK, Lefkowitz EJ, Kremmidiotis G, D'Eustachio P, Beier DR, Guay-Woodford LM. Cystin, a novel ciliaassociated protein, is disrupted in the cpk mouse model of polycystic kidney disease. J Clin Invest 109:533-540, 2002.

4. Nachury MV, Loktev AV, Zhang Q, Westlake CJ, Peranen J, Merdes A, Slusarski DC, Scheller RH, Bazan JF, Sheffield VC, Jackson PK. A core complex of BBS proteins cooperates with the GTPase Rab8 to promote ciliary membrane biogenesis. Cell 129:1201-1213, 2007.

5. Yoder BK. Role of primary cilia in the pathogenesis of polycystic kidney disease. J Am Soc Nephrol 18:1381-1388, 2007.

6. Masyuk AI, Masyuk TV, Splinter PL, Huang BQ, Stroope AJ, LaRusso NF. Cholangiocyte cilia detect changes in luminal fluid flow and transmit them into intracellular $\mathrm{Ca} 2+$ and cAMP signaling. Gastroenterology 131:911-920, 2006.

7. Pan J, Snell W. The primary cilium: keeper of the key to cell division. Cell 129:1255-1257, 2007.
8. Praetorius HA, Spring KR. The renal cell primary cilium functions as a flow sensor. Curr Opin Nephrol Hypertens 12:517-520, 2003.

9. Praetorius HA, Spring KR. Removal of the MDCK cell primary cilium abolishes flow sensing. J Membr Biol 191:69-76, 2003.

10. Praetorius HA, Spring KR. Bending the MDCK cell primary cilium increases intracellular calcium. J Membr Biol 184:71-79, 2001.

11. Hanaoka K, Guggino WB. cAMP regulates cell proliferation and cyst formation in autosomal polycystic kidney disease cells. J Am Soc Nephrol 11:1179-1187, 2000.

12. Sutters M, Yamaguchi T, Maser RL, Magenheimer BS, St John PL, Abrahamson DR, Grantham JJ, Calvet JP. Polycystin-1 transforms the cAMP growth-responsive phenotype of M-1 cells. Kidney Int 60:484 494, 2001.

13. Yamaguchi T, Wallace DP, Magenheimer BS, Hempson SJ, Grantham JJ, Calvet JP. Calcium restriction allows cAMP activation of the BRaf/ERK pathway, switching cells to a cAMP-dependent growthstimulated phenotype. J Biol Chem 279:40419-40430, 2004.

14. Ye M, Grantham JJ. The secretion of fluid by renal cysts from patients with autosomal dominant polycystic kidney disease. N Engl J Med 329: 310-313, 1993.

15. Davidow CJ, Maser RL, Rome LA, Calvet JP, Grantham JJ. The cystic fibrosis transmembrane conductance regulator mediates transepithelial fluid secretion by human autosomal dominant polycystic kidney disease epithelium in vitro. Kidney Int 50:208-218, 1996.

16. Grantham JJ, Ye M, Gattone VH 2nd, Sullivan LP. In vitro fluid secretion by epithelium from polycystic kidneys. J Clin Invest 95:195202, 1995.

17. Mangoo-Karim R, Ye M, Wallace DP, Grantham JJ, Sullivan LP. Anion secretion drives fluid secretion by monolayers of cultured human polycystic cells. Am J Physiol 269:F381-388, 1995.

18. Nichols MT, Gidey E, Matzakos T, Dahl R, Stiegmann G, Shah RJ, Grantham JJ, Fitz JG, Doctor RB. Secretion of cytokines and growth factors into autosomal dominant polycystic kidney disease liver cyst fluid. Hepatology 40:836-846, 2004.

19. Cohn JA, Strong TV, Picciotto MR, Nairn AC, Collins FS, Fitz JG. Localization of the cystic fibrosis transmembrane conductance regulator in human bile duct epithelial cells. Gastroenterology 105:1857-1864, 1993.

20. Fitz JG, Basavappa S, McGill J, Melhus O, Cohn JA. Regulation of membrane chloride currents in rat bile duct epithelial cells. J Clin Invest 91:319-328, 1993.

21. Doctor RB, Johnson S, Brodsky KS, Amura CR, Gattone V, Fitz JG. Regulated ion transport in mouse liver cyst epithelial cells. Biochim Biophys Acta 1772:345-354, 2007.

22. Kirkland JG, Cottrell GS, Bunnett NW, Corvera CU. Agonists of protease-activated receptors 1 and 2 stimulate electrolyte secretion from mouse gallbladder. Am J Physiol Gastrointest Liver Physiol 293:G335346, 2007.

23. Ricker JL, Gattone VH 2nd, Calvet JP, Rankin CA. Development of autosomal recessive polycystic kidney disease in BALB/c-cpk/cpk mice. J Am Soc Nephrol 11:1837-1847, 2000.

24. Gattone VH 2nd, Wang X, Harris PC, Torres VE. Inhibition of renal cystic disease development and progression by a vasopressin V2 receptor antagonist. Nat Med 9:1323-1326, 2003.

25. Torres VE, Wang X, Qian Q, Somlo S, Harris PC, Gattone VH 2nd. Effective treatment of an orthologous model of autosomal dominant polycystic kidney disease. Nat Med 10:363-364, 2004.

26. Harding MA, Gattone VH 2nd, Grantham JJ, Calvet JP. Localization of overexpressed c-myc mRNA in polycystic kidneys of the cpk mouse. Kidney Int 41:317-325, 1992.

27. Li JB, Gerdes JM, Haycraft CJ, Fan Y, Teslovich TM, May-Simera H, Li H, Blacque OE, Li L, Leitch CC, Lewis RA, Green JS, Parfrey PS, Leroux MR, Davidson WS, Beales PL, Guay-Woodford LM, Yoder BK, Stormo GD, Katsanis N, Dutcher SK. Comparative genomics 
identifies a flagellar and basal body proteome that includes the BBS5 human disease gene. Cell 117:541-552, 2004.

28. Pazour GJ, Agrin N, Leszyk J, Witman GB. Proteomic analysis of a eukaryotic cilium. J Cell Biol 170:103-113, 2005.

29. Yoder BK, Hou X, Guay-Woodford LM. The polycystic kidney disease proteins, polycystin-1, polycystin-2, polaris, and cystin, are colocalized in renal cilia. J Am Soc Nephrol 13:2508-2516, 2002.

30. Hildebrandt F, Zhou W. Nephronophthisis-associated ciliopathies. J Am Soc Nephrol 18:1855-1871, 2007.

31. Masyuk TV, Huang BQ, Ward CJ, Masyuk AI, Yuan D, Splinter PL, Punyashthiti R, Ritman EL, Torres VE, Harris PC, LaRusso NF.
Defects in cholangiocyte fibrocystin expression and ciliary structure in the PCK rat. Gastroenterology 125:1303-1310, 2003.

32. Guay-Woodford LM, Green WJ, Lindsey JR, Beier DR. Germline and somatic loss of function of the mouse cpk gene causes biliary ductal pathology that is genetically modulated. Hum Mol Genet 9:769-778, 2000.

33. Becker JH, Willis JS. Properties of Na-K pump in primary cultures of kidney cells. J Cell Physiol 99:427-439, 1979.

34. Masyuk TV, Masyuk AI, Torres VE, Harris PC, Larusso NF. Octreotide inhibits hepatic cystogenesis in a rodent model of polycystic liver disease by reducing cholangiocyte adenosine $3^{\prime}, 5^{\prime}$-cyclic monophosphate. Gastroenterology 132:1104-1116, 2007. 